\title{
Seasonal and Geographic Variation in Adverse Event Reporting
}

\author{
Osvaldo Marrero $^{1} \cdot$ Eric Y. Hung ${ }^{2} \cdot$ Manfred Hauben ${ }^{2,3}$
}

Published online: 11 June 2016

(C) The Author(s) 2016. This article is published with open access at Springerlink.com

\begin{abstract}
Background Many illnesses demonstrate seasonal and geographic variations. Pharmacovigilance is unique among public health surveillance systems in terms of the clinical diversity of the events under surveillance. Since many pharmacovigilance signal detection methodologies are geared towards looking for increased frequency of spontaneous adverse drug event (ADE) reporting over variable time frames, seasonality of ADEs may have implications for signal detection.

Objective The aim of this study was to investigate whether a set of illnesses that might be expected to display seasonality in general, did so when spontaneously reported as ADEs.

Methods We performed our analysis with the publically available US FDA Adverse Event Reporting System (FAERS) data. We selected a convenience sample of events possibly triggered by seasonal factors (hypothermia, Raynaud's phenomenon, photosensitivity reaction, heat exhaustion, heat stroke, and sunburn) and events for which previous literature experience suggests seasonality (anencephaly and interstitial lung disease). Our statistical procedures can be explained in terms of a simple physicogeometric setting: the unit circle divided into 6 (semiannual sinusoidal) or 12 (annual sinusoidal) arcs. When reporting frequencies (weights) are more or less evenly distributed across months, the center of mass of the circle would not be significantly displaced from the origin
\end{abstract}

Eric Y. Hung

eric.hung@pfizer.com

1 Department of Mathematics and Statistics, Villanova University, Villanova, PA, USA

2 Pfizer Inc., New York, NY, USA

3 New York University Medical Center, New York, NY, USA
$(0,0)$. Distinct seasonal patterns will significantly displace the center of mass of the circle.

Results Various patterns of seasonality were identified for some, but not all, events and region-event pairs. USA displayed the most instances of seasonality. Scandinavia did not display seasonality for any events. Seasonality was usually annual sinusoidal. Possible explanations for failure to observe seasonality are briefly considered.

Conclusions Understanding seasonality of spontaneous ADE reporting may have public health policy and research implications and may mitigate false-positive and missed true-positive pharmacovigilance signals. More systematic study of seasonality of spontaneous ADE reporting, including additional events with more or less biological rationale for seasonality, is a logical extension of this analysis.

\section{Key Points}

Adverse events may display seasonal and geographic reporting variations.

Awareness of these patterns may improve the interpretation of outputs from signal detection exercises.

Knowledge of adverse event seasonality may provide opportunities for public health intervention.

\section{Introduction}

Various illnesses have been reported to display seasonal variation. Some of these have obvious intuitive plausibility (e.g., heat exhaustion and other temperature- or weather- 
related conditions, vector-borne conditions affected by weather and light, events affected by seasonal behavior including food consumption, travel, alcohol consumption, and/or recreational activities). For others it might not be as obvious, and for some no clear explanation is apparent (e.g., Kawasaki disease [1], atrial fibrillation [2]).

A principal concern of pharmacovigilance is the ongoing monitoring of spontaneous reports of illnesses for which the reporter suspects a causal relationship with one or more drugs, medical devices, or biologics. Pharmacovigilance is rather unique as far as monitoring/surveillance programs go by virtue of the extreme diversity of the clinical and quantitative phenotypes of illnesses under surveillance. Therefore, we were curious whether seasonality of medical illnesses may extend into the reporting of these illnesses as suspected adverse drug reactions. Such patterns, if present, could have implications for both public health policy and adverse event monitoring, specifically the interpretation of potential pharmacovigilance signals based on changes in spontaneous adverse event (AE) reporting frequency. For example, some traditional approaches to pharmacovigilance signal detection are based on detecting increased AE reporting frequency. Depending on operational details, a seasonal increase in $\mathrm{AE}$ reporting could be attributed to a drug. This may be especially true for new or infrequently monitored drugs which may not have accumulated a sufficient corpus of $\mathrm{AE}$ reports over time to clearly demonstrate seasonality.

The objective of the current analysis is to investigate whether various illnesses that are reported in the context of a suspect adverse drug reaction display seasonal and/or geographic variations. We analyze data from a number of conditions that can be partitioned into three classes. First we consider the ones that may be triggered by cold weather; next those that are associated with warm weather; and then those adverse drug events (ADEs) for which previous literature or experience suggest seasonality, even if there is not an obvious intuitive justification or expectation of seasonality.

\section{Methods}

\subsection{The Data}

The FDA maintains the Adverse Event Reporting System (FAERS), a database that contains information related to post-marketing medication error reports and safety surveillance as well as individually reported adverse events submitted by healthcare providers and consumers themselves, which may include patients, family members, or lawyers. The FAERS is evaluated and overseen by clinical reviewers in the Center for Drug Evaluation and Research
(CDER) and the Center for Biologics Evaluation and Research (CBER). The CDER and CBER monitor these reports for any potential safety concerns after the drug is initially approved by the FDA [3]. Adverse events are recorded in FAERS using the Medical Dictionary for Regulatory Affairs (MedDRA Version 15.0) Preferred Terms (PTs) [4, 5]. PTs are intended to represent a single medical concept and linked with broader Higher-Level Terms (HLTs), Higher-Level Group Terms (HLGTs), System Organ Classes (SOCs), and narrower groupings (reported terms) in a hierarchical structure.

Gathered from the public release of the FAERS database, the data are for the period 1997-2011, all years inclusively. Separately for each region and each condition, the data were pooled into twelve monthly totals. The regions are Japan, Scandinavia, and the US. Our selection of regions was based on the fact that these areas have well developed pharmacovigilance systems and, while a convenience sample, these regions were otherwise sufficiently diverse to allow exploration of seasonal and geographic variation.

\subsection{Events Selected}

For purposes of this pilot study, we performed a 2-step convenience sample. First we selected a convenience sample of medical events that may be triggered by seasonal factors. Then we identified the closest matching MedDRA PTs by manual inspection of MedDRA. For cold weather, we consider the PTs hypothermia and Raynaud's phenomenon. In the category of events that are associated with warm weather, we study the PTs photosensitivity reaction, heat exhaustion, heat stroke, and sunburn. In the last set-those ADEs for which previous literature or experience suggest seasonality, even if there is not an obvious intuitive justification or expectation of seasonality - we consider the PTs anencephaly and interstitial lung disease; for instance, in the studies by Edwards and Marrero [6-8], anencephaly was found to exhibit seasonal variation, and Olson et al. [9] found that mortality from pulmonary fibrosis is greatest in the winter.

\subsection{Statistical Analyses}

Statistical tests of seasonality include $\chi^{2}$ and harmonic analysis in which the monthly pattern of observations is approximated using sine and cosine functions. We use a form of harmonic analysis. The original sources for the specific form of harmonic analysis are Marrero [7] for the singlegroup case and Marrero [10] for the multigroup case. We chose this method because a simulation study [7] shows that it performs better than another form of harmonic analysis known as Edwards's test [6] and the chi-square test. In particular, the study [7] reveals that our method has the correct type I error rate for sample sizes as low as 15. Edwards's test 


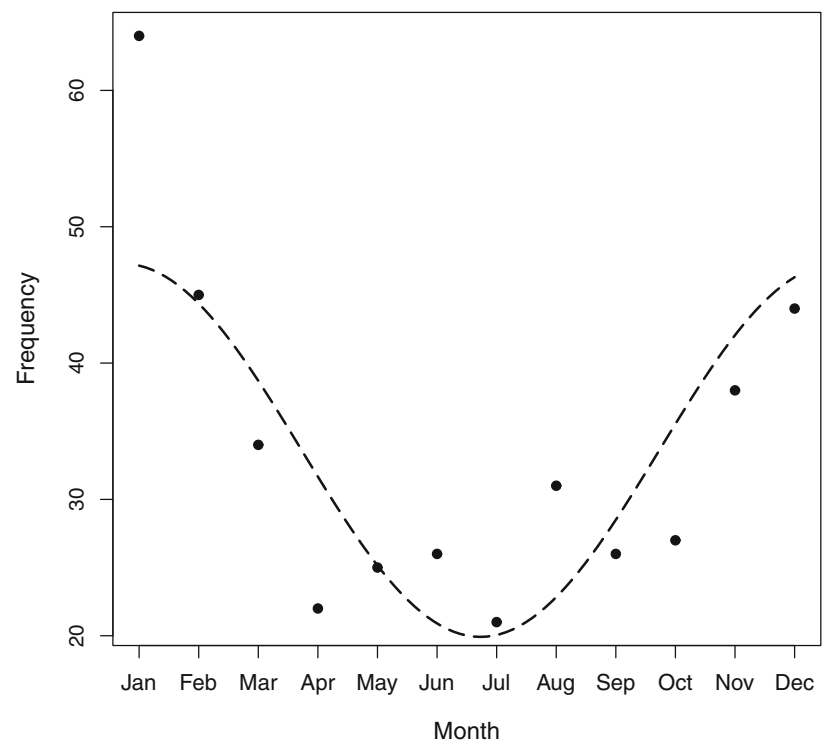

Fig. 1 Diabetes mellitus: data and model

has an unacceptably high type I error rate with small sample sizes. Moreover, in Marrero [7] it is also shown that our procedure is often $25 \%$ more powerful than the chi-square test, and such gain can be as high as $40 \%$.

In the medical sciences, three commonly seen seasonal patterns are annual sinusoidal, semiannual sinusoidal, and annual unimodal. Our method is adequate and can produce statistically significant results for all three of these patterns. As examples, our procedure has been successfully used to demonstrate seasonality for diabetes mellitus diagnoses [11], whose annual sinusoidal pattern is shown in Fig. 1; Crohn's disease onset of symptoms [11], whose semiannual sinusoidal pattern is shown in Fig. 2; and the cases of extrahepatic biliary atresia (EHBA) [12], whose rectangular annual unimodal pattern is shown in Fig. 3. Remarkably, the EHBA dataset is a concrete example of the method's high power: a statistically significant result was obtained with just 30 observations.

We used the number of spontaneous reports per month as our primary outcome metric. The null hypothesis is that of uniformity: the probability that an event occurs is the same for all the months. The alternative hypothesis is unrestricted (i.e., it is unnecessary to specify the shape of the deviation from uniformity) and this is an advantage of these methods; however, in practice, investigators specify a priori a pattern for the alternative hypothesis.

\section{Results}

Of eight events examined, four didn't have enough data for the multigroup procedure, which produced statistically significant results for three of the other four events. Of 24

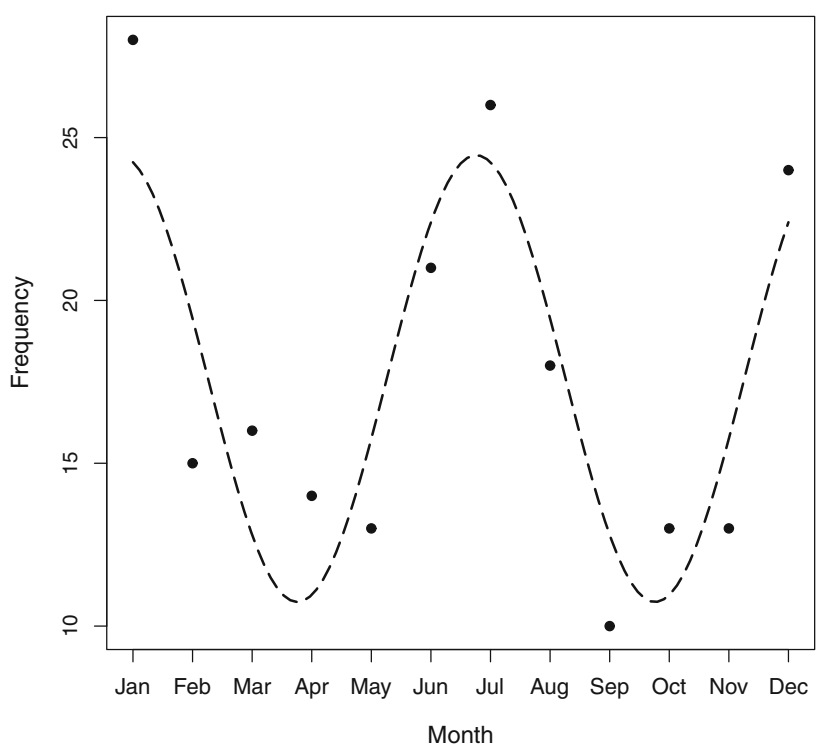

Fig. 2 Crohn's disease: data and model

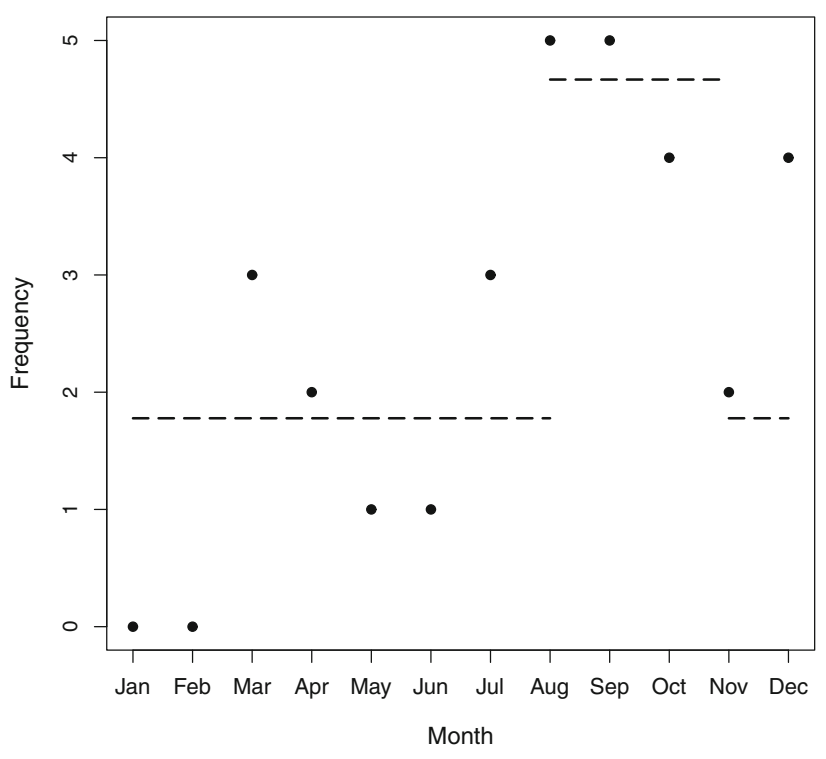

Fig. 3 Extrahepatic biliary atresia: data and model

region-event pairs, seven displayed seasonal reporting patterns for the single-group procedure (ten not performed due to limited data). The detailed findings are now presented.

\subsection{Hypothermia}

Separately by region, the data for Japan, Scandinavia, and the US were pooled into twelve monthly totals that are shown in Table 1.

First we applied the multigroup procedure [10] to simultaneously test for seasonality in all three regions. For 
Table 1 FAERS data (1997-2011) monthly totals for events possibly triggered by seasonal factors or for which previous literature experience suggests seasonality, by region

\begin{tabular}{|c|c|c|c|c|c|c|c|c|c|c|c|c|}
\hline Region & Jan & Feb & Mar & Apr & May & Jun & Jul & Aug & Sep & Oct & Nov & Dec \\
\hline \multicolumn{13}{|l|}{ Hypothermia } \\
\hline Japan & 16 & 29 & 20 & 13 & 8 & 9 & 6 & 11 & 6 & 7 & 7 & 15 \\
\hline Scandinavia & 8 & 4 & 4 & 5 & 4 & 1 & 5 & 6 & 3 & 4 & 3 & 5 \\
\hline USA & 30 & 41 & 34 & 38 & 32 & 27 & 41 & 22 & 51 & 33 & 38 & 47 \\
\hline \multicolumn{13}{|c|}{ Raynaud's phenomenon } \\
\hline Japan & 2 & 5 & 6 & 0 & 1 & 1 & 0 & 2 & 2 & 1 & 3 & 2 \\
\hline Scandinavia & 0 & 1 & 1 & 5 & 4 & 2 & 1 & 3 & 5 & 3 & 2 & 2 \\
\hline USA & 29 & 25 & 42 & 31 & 18 & 32 & 25 & 30 & 26 & 33 & 36 & 36 \\
\hline \multicolumn{13}{|c|}{ Photosensitivity reaction } \\
\hline Japan & 3 & 2 & 8 & 7 & 14 & 13 & 11 & 6 & 12 & 7 & 4 & 4 \\
\hline Scandinavia & 1 & 4 & 4 & 5 & 4 & 8 & 4 & 7 & 4 & 1 & 3 & 5 \\
\hline USA & 82 & 140 & 99 & 70 & 65 & 102 & 126 & 152 & 119 & 91 & 116 & 167 \\
\hline \multicolumn{13}{|c|}{ Heat exhaustion } \\
\hline Japan & 0 & 0 & 0 & 0 & 0 & 1 & 0 & 1 & 0 & 3 & 0 & 0 \\
\hline Scandinavia & 0 & 0 & 0 & 0 & 0 & 0 & 1 & 0 & 0 & 0 & 0 & 0 \\
\hline USA & 3 & 3 & 11 & 2 & 8 & 13 & 15 & 19 & 14 & 5 & 13 & 5 \\
\hline \multicolumn{13}{|l|}{ Heat stroke } \\
\hline Japan & 0 & 0 & 0 & 0 & 0 & 0 & 2 & 0 & 4 & 1 & 2 & 0 \\
\hline Scandinavia & 0 & 0 & 0 & 0 & 1 & 0 & 0 & 0 & 0 & 0 & 0 & 0 \\
\hline USA & 6 & 2 & 5 & 3 & 8 & 19 & 17 & 18 & 21 & 9 & 6 & 11 \\
\hline \multicolumn{13}{|l|}{ Sunburn } \\
\hline Japan & 0 & 0 & 0 & 0 & 0 & 1 & 0 & 0 & 0 & 0 & 1 & 0 \\
\hline Scandinavia & 0 & 0 & 0 & 1 & 1 & 1 & 0 & 0 & 0 & 0 & 0 & 0 \\
\hline USA & 27 & 31 & 27 & 28 & 28 & 49 & 53 & 84 & 47 & 40 & 42 & 83 \\
\hline \multicolumn{13}{|l|}{ Anencephaly } \\
\hline Japan & 1 & 1 & 1 & 0 & 0 & 0 & 1 & 1 & 0 & 0 & 1 & 0 \\
\hline Scandinavia & 0 & 1 & 1 & 0 & 0 & 0 & 1 & 0 & 2 & 0 & 1 & 1 \\
\hline USA & 3 & 4 & 1 & 1 & 4 & 1 & 2 & 4 & 6 & 2 & 3 & 5 \\
\hline \multicolumn{13}{|c|}{ Interstitial lung disease } \\
\hline Japan & 197 & 242 & 290 & 265 & 246 & 321 & 268 & 284 & 279 & 268 & 280 & 350 \\
\hline Scandinavia & 7 & 9 & 7 & 4 & 4 & 3 & 14 & 5 & 3 & 6 & 6 & 4 \\
\hline USA & 70 & 62 & 90 & 67 & 74 & 89 & 107 & 95 & 90 & 110 & 98 & 144 \\
\hline
\end{tabular}

FAERS US FDA Adverse Event Reporting System each region, the null hypothesis was that of uniformity, and the alternative hypothesis was that the data follow an annual sinusoidal pattern with peak in winter and trough in summer. The result was statistically significant $(p=0.000028)$. As is evident from Fig. 4 , the three regions appear to follow different patterns. Further analyses were, therefore, conducted separately by region, following the method developed by Marrero [7], and using the same hypotheses.

The data from Japan produced a statistically significant result $(p=0.0000011)$. However, the data from Scandinavia were not statistically significant $(p=0.6492)$, and neither were the data from the US $(p=0.2907)$. This agrees with what is shown in Fig. 4, where we see that the descriptive annual sinusoidal models for Scandinavia and the US are nearly flat, yet there is some oscillation in the
US model, but not enough to be significant, and, moreover, the model does not fit the data well. For Japan, however, an annual sinusoidal model fits the data well.

\subsection{Raynaud's Phenomenon}

Separately by region, the data from Japan, Scandinavia, and the US were pooled into twelve monthly totals that are shown in Table 1.

We applied the multigroup procedure [10] to simultaneously test for seasonality in all three regions. For each region, the null hypothesis was that of uniformity. The alternative hypothesis was that the data follow an annual sinusoidal pattern with peak in winter and trough in summer. The result was not statistically significant $(p=0.0921)$. 
The Japan data contributed the most $(55 \%)$ to the multigroup test-statistic value, and the contributions from the other regions were about equal. For that reason, we decided to test the Japan data alone for annual seasonality; the result was close to being significant $(p=0.0505)$. In Fig. 5, we note that the data from the US show variability about the sinusoidal model, especially earlier in the year, and the Scandinavia data are essentially flat, which helps to explain the nonsignificance for these two regions. For Japan, the data appear reasonably faithful to the sinusoidal model, and that agrees with the low $p$ value. Probably one

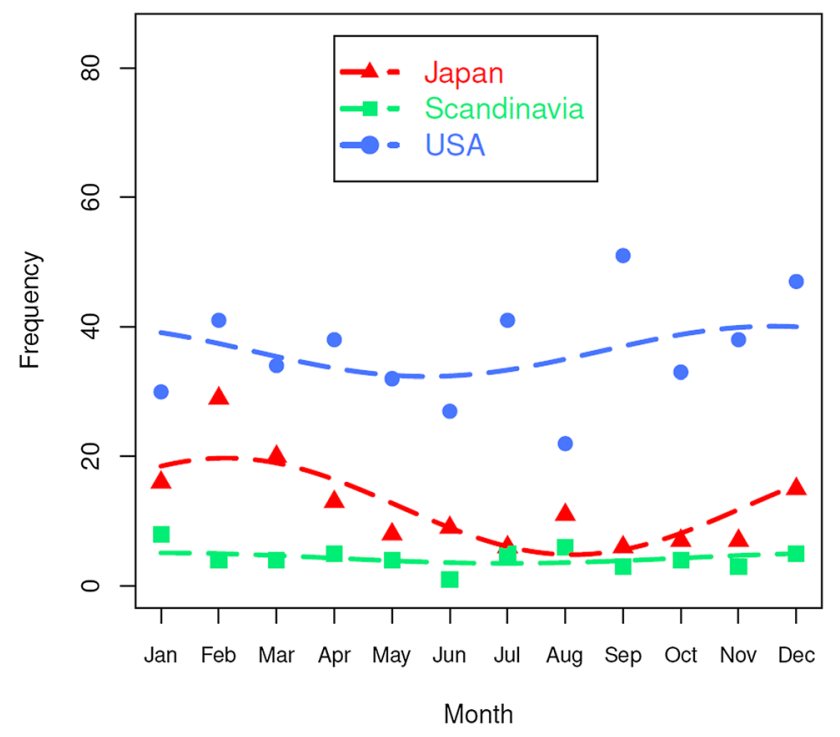

Fig. 4 Hypothermia: FAERS data (1997-2011) and models. FAERS US FDA Adverse Event Reporting System

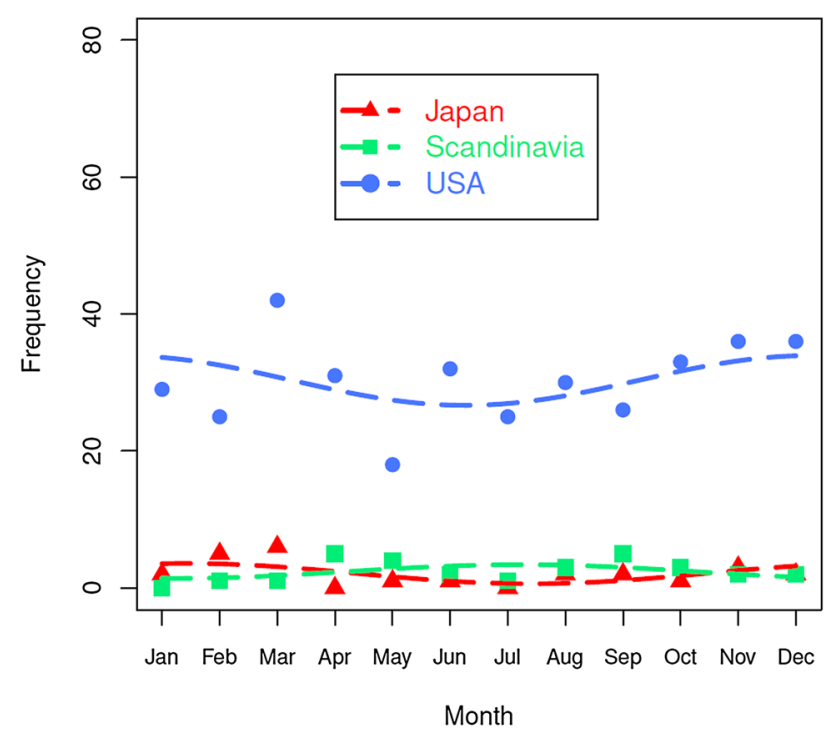

Fig. 5 Raynaud's phenomenon: FAERS data (1997-2011) and models. FAERS US FDA Adverse Event Reporting System the most often cited limitations of data from a spontaneous reporting system (SRS), underreporting may also contribute to the nonsignificance.

\subsection{Photosensitivity Reaction}

Separately by region, the data from Japan, Scandinavia, and the US were pooled into twelve monthly totals that are shown in Table 1.

First we applied the multigroup procedure [10] to simultaneously test for seasonality in all three regions. For each region, the null hypothesis was that of uniformity. The alternative hypothesis for Japan and Scandinavia was that the data follow an annual sinusoidal pattern with peak in summer and trough in winter, and for the US that the data follow a semiannual sinusoidal pattern with peaks in winter and summer, and troughs in the other seasons. The result was statistically significant $(p=0.000000007)$. As is evident from Fig. 6, the three regions appear to follow different patterns. Further analyses were, therefore, conducted separately by region, following the method developed by Marrero [7], and using the same hypotheses.

The data from Japan produced a statistically significant result $(p=0.0003)$, and so did the data from the US $(p=0.00000058)$. However, the data from Scandinavia were not statistically significant $(p=0.1372)$. This agrees with what is shown in Fig. 6, where we note the following. For Japan, an annual sinusoidal model fits the data well. For the US, a semiannual sinusoidal model is adequate, but some data points do not conform well to the model. The data for Scandinavia are nearly constant.

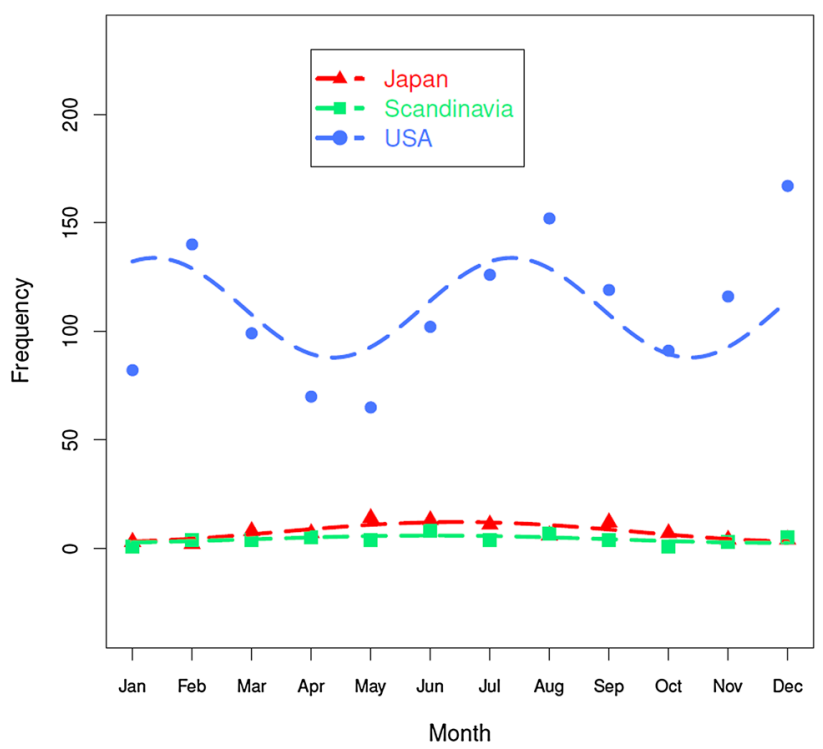

Fig. 6 Photosensitivity reaction: FAERS data (1997-2011) and models. FAERS US FDA Adverse Event Reporting System 


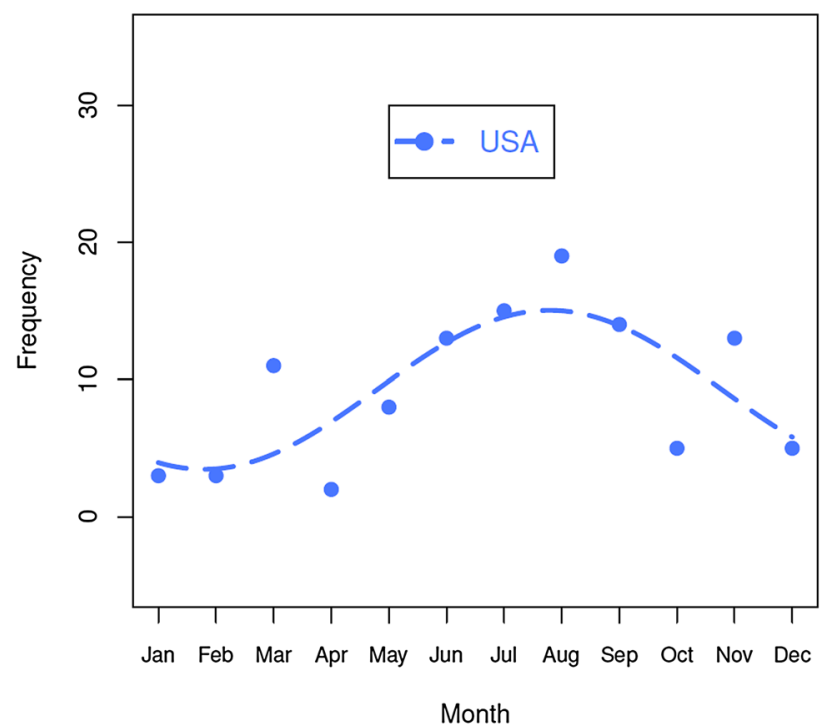

Fig. 7 Heat exhaustion: FAERS data (1997-2011) and model. FAERS US FDA Adverse Event Reporting System

\subsection{Heat Exhaustion}

We only considered the data for the US because there were too few observations for the other two regions: five observations for Japan and one observation for Scandinavia. The data for the US were pooled into twelve monthly totals that are shown in Table 1.

We applied the single-group procedure [7] to test the US data for seasonality. The null hypothesis was that of uniformity. The alternative hypothesis was that the data follow an annual sinusoidal pattern with peak in summer and trough in winter. The result was statistically significant $(p=0.0000194)$. This agrees with what is shown in Fig. 7, where we see that the descriptive annual sinusoidal model for the US shows annual sinusoidal variation, with a peak in mid August and trough in mid February; the model fits the data well.

\subsection{Heat Stroke}

We only considered the data for the US because there were too few observations for the other two regions: nine observations for Japan and one observation for Scandinavia. The data for the US were pooled into twelve monthly totals that are shown in Table 1.

We applied the single-group procedure [7] to test the US data for seasonality. The null hypothesis was that of uniformity. The alternative hypothesis was that the data follow an annual sinusoidal pattern with peak in summer and trough in winter. The result was statistically significant $(p=0.000000033)$. This agrees with what is shown in Fig. 8, where we see that the descriptive annual sinusoidal

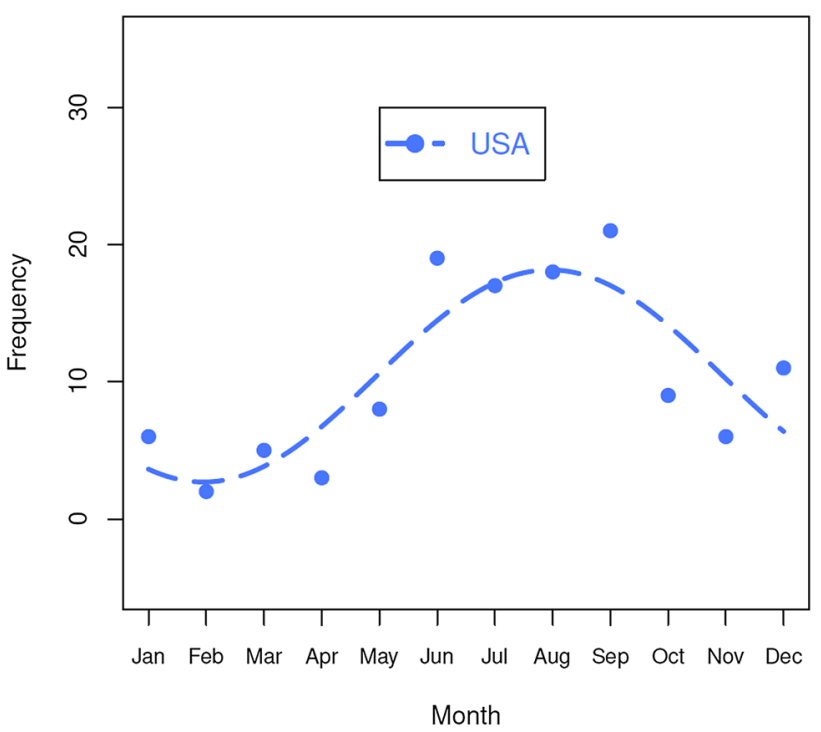

Fig. 8 Heat stroke: FAERS data (1997-2011) and model. FAERS US FDA Adverse Event Reporting System

model for the US shows annual sinusoidal variation, with a peak in mid August and trough in mid February; the model fits the data well.

\subsection{Sunburn}

We only considered the data for the US because there were too few observations for the other two regions: two observations for Japan and three observations for Scandinavia. The data for the US were pooled into twelve monthly totals that are shown in Table 1.

We applied the single-group procedure [7] to test the US data for seasonality. The null hypothesis was that of uniformity. The alternative hypothesis was that the data follow an annual sinusoidal pattern with peak in summer and trough in winter. The result was statistically significant $(p=0.000000033)$. It is clear from Fig. 9 that an annual sinusoidal model does not fit the data well. From January to July, the data appear to follow an annual sinusoidal pattern with low points in January through May, and then increases as the weather gets warmer in June and July. However, the data from August through December are best described by a U-shaped model that has maxima in August and December, and a nadir in October.

\subsection{Anencephaly}

We only considered the data for the US because there were too few observations for the other two regions: six observations for Japan and seven observations for Scandinavia. The data for the US were pooled into twelve monthly totals that are shown in Table 1. 


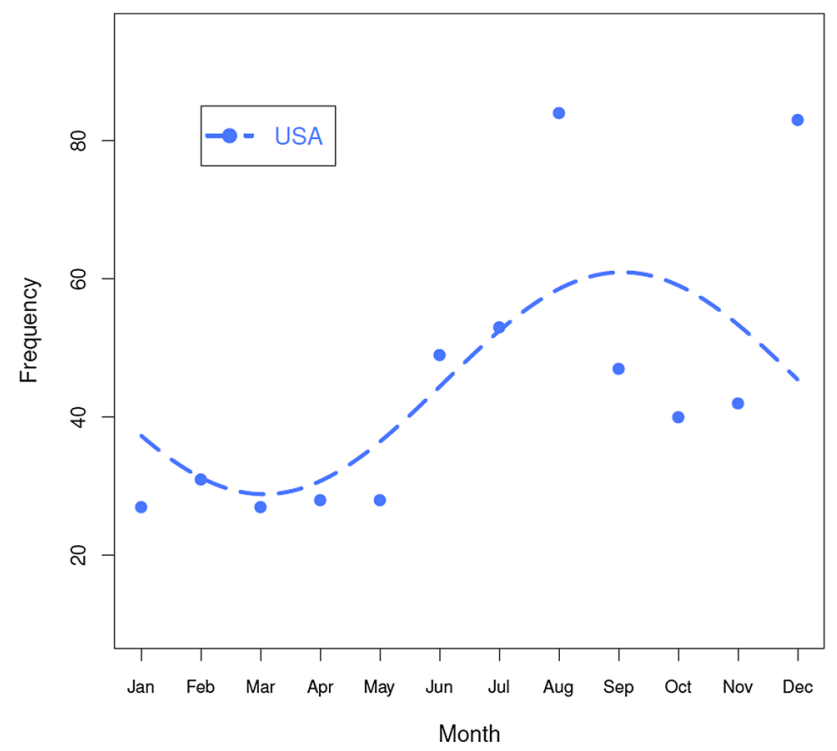

Fig. 9 Sunburn: FAERS data (1997-2011) and model. FAERS US FDA Adverse Event Reporting System

We applied the single-group procedure [7] to test the US data for seasonality. The null hypothesis was that of uniformity. The alternative hypothesis was that the data follow an annual sinusoidal pattern with peak in winter and trough in summer. The result was not statistically significant $(p=0.2952)$. In Fig. 10 we see that both the data and the corresponding descriptive model are essentially flat.

\subsection{Interstitial Lung Disease}

Separately by region, the data for Japan, Scandinavia, and the US were pooled into twelve monthly totals that are shown in Table 1.

First we applied the multigroup procedure [10] to simultaneously test for seasonality in all three regions. For each region, the null hypothesis was that of uniformity, and the alternative hypothesis was that the data follow an annual sinusoidal pattern with peak in winter and trough in summer. The result was statistically significant $(p=0.000269)$. As is evident from Fig. 11, the three regions appear to follow different patterns. Further analyses were, therefore, conducted separately by region, following the method developed by Marrero [7], and using the same hypotheses.

The data from the US produced a statistically significant result $(p=0.000024)$. However, the data from Japan were not statistically significant $(p=0.1197)$, and so were the data from Scandinavia $(p=0.9777)$.

The individual $p$ values agree with what is shown in Fig. 11, where we see that the descriptive annual sinusoidal model for the US fits the data well, with maximum

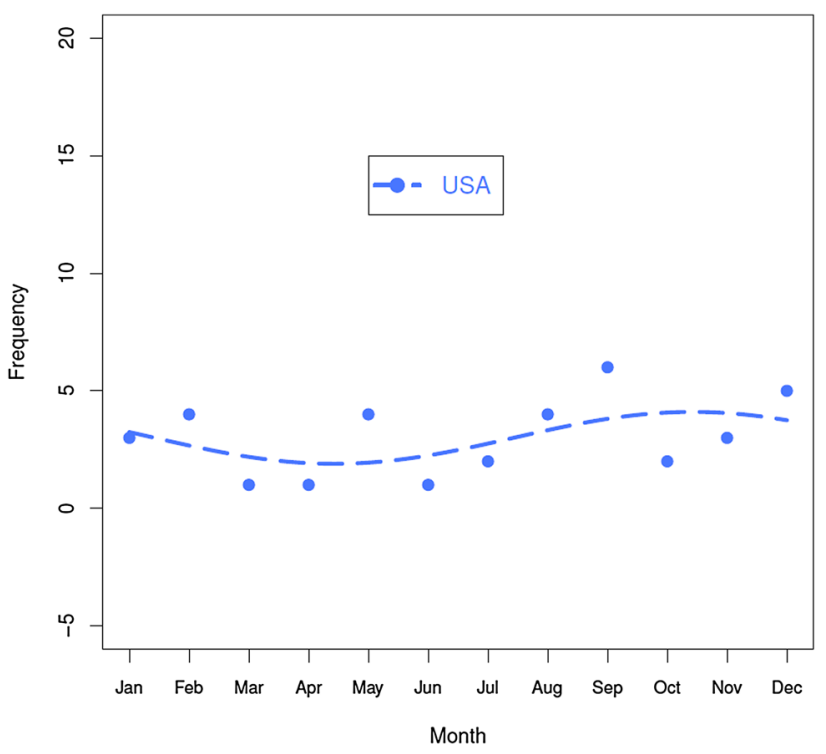

Fig. 10 Anencephaly: FAERS data (1997-2011) and model. FAERS US FDA Adverse Event Reporting System

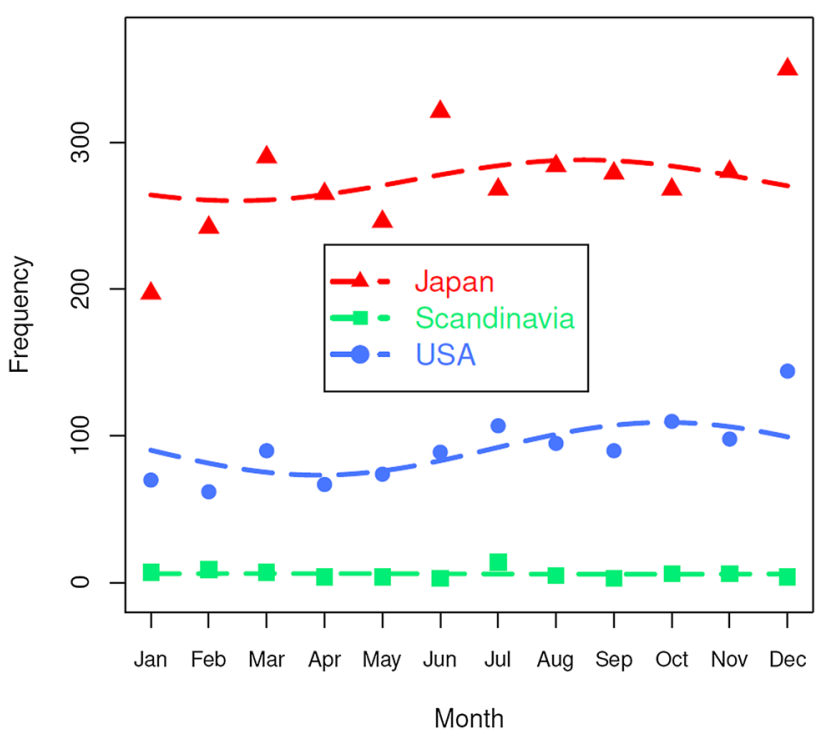

Fig. 11 Interstitial lung disease: FAERS data (1997-2011) and models. FAERS US FDA Adverse Event Reporting System

spontaneous reporting frequency in mid October and minimum spontaneous reporting frequency in mid April. The data from Japan generally appear to follow an annual sinusoidal model with higher data on average than that of the US, but there are three aberrant observations-January, June, and December-that do not conform well to the model; this lack of fit, of course, helps to increase the $p$ value. The data from Scandinavia are essentially flat.

Our findings by region are summarized in Table 2 . 
Table 2 Results summary of the seasonality analysis

\begin{tabular}{llll}
\hline & Nonsignificant & Annual sinusoidal & Semiannual sinusoidal \\
\hline Multigroup & Raynaud's phenomenon & $\begin{array}{l}\text { Hypothermia } \\
\text { Interstitial lung disease }\end{array}$ & \\
Single group & & Hypothermia \\
Japan & Raynaud's phenomenon & Photosensitivity & \\
Scandinavia & Interstitial lung disease & & \\
& Hypothermia & & \\
& Photosensitivity & Photosensitivity \\
USA & Interstitial lung disease & Heat exhaustion & \\
& Hypothermia & Heat stroke & \\
& Anencephaly & Sunburn & \\
& & Interstitial lung disease & \\
& &
\end{tabular}

\section{Discussion}

We have found that the spontaneous reporting of selected adverse drug events display seasonal/cyclic variation and that these variations occur more or less in relation to geographic locale. Of eight events examined, four didn't have enough data for multigroup procedure, which returned statistically significant results for three of the other four events. Of 24 region-event pairs, seven displayed seasonal reporting patterns and ten were not analyzed because of limited data. Various patterns of seasonality were identified for some, but not all, events and region-event pairs. When seasonality was observed, it was usually annual sinusoidal. The US displayed the most instances of seasonality. Scandinavia did not display seasonality for any events. One or more heat-/light-related events displayed seasonality in both Japan and the US, while cold-related events displayed seasonality only in Japan. Of our third class of events, those for which literature suggests seasonality despite no obvious intuitive justification, only interstitial lung disease displayed seasonality, and it was only in the US. For the US, multiple clinically similar heat-/light-related events displayed seasonality. Other groups of events did not show similarly consistent reporting patterns.

We can only speculate about why some events and region-event pairs did not display expected patterns (based on the biological plausibility arguments or actual data for the events when not linked to drug administration). Possible hypotheses to explain these instances include geographic differentials in inter-seasonal temperature gradients (in geographic regions otherwise at comparable latitudes), differential population mobility/travel, genetic adaptation to environment, public health support infrastructure, cultural norms, and the intensity of surveillance. It is likely that for some events, univariate model of seasonality is an over-simplification. For example, intuition suggests increased photosensitivity during warmer seasons when people are outdoors and the days are longer. Yet other factors, such as cloud cover, may confound this relationship, and would not be directly accommodated in our models [13]. Additionally, numerous factors influence spontaneous reporting, and these may distort the link between actual occurrence and reporting.

Our findings, while intrinsically interesting, may also potentially have practical implications for pharmacovigilance and public health policy. First, if it is verified that some of the geographic gradients in overall reporting and/or seasonality that we observed with spontaneous reporting are in part a function of public health education as, for example, we speculated for the heat exhaustion and heat stroke events, then this suggests possibilities for public health interventions. Second, in the domain of pharmacovigilance, surveillance for potential signals of novel adverse effects of drugs, devices, and biologicals may entail monitoring spontaneous reporting frequencies or various functions of these frequencies. When such potential signals are identified based on relatively short monitoring time windows/comparison periods, the possibility of a false-positive signal due to seasonality or other cyclic variation may be considered and evaluated prior to full signal evaluation. This could help focus finite pharmacovigilance resources by reducing time and energy spent on false-positive findings. This may be especially pertinent for adverse events that do not have an obvious intuitive rationale to expect seasonality/cyclic variation. It is even theoretically possible that seasonal variation could obscure a signal (i.e., a false negative).

There are limitations to our analysis. The enormous limitations of SRS databases, which are well documented and which have been alluded to in one form or another in this paper, prevent making any inferences about the underlying population risks of these events or the temporal variation of these risks. When comparing overall reporting between international regions, we note that the FDA criteria for inclusion of spontaneous reports submitted to them 
become a sort of filter, so the FAERS database may represent a biased sample with regards to international reports; the bias may be in part related to the novelty and seriousness of the event. A corollary of these limitations is that many of our proposed explanations remain hypothetical. We examined individual MedDRA PTs. More detailed future analyses might fruitfully use higher levels of the MedDRA hierarchy, or pool conceptually related individual PTs to increase effective sample size. This might allow analysis for geographic area-event pairs for which there were not enough data available for this first-pass analysis. It would also provide a check on the robustness of findings, since one would expect that events that are sufficiently related to share a common pathophysiological basis should display similar seasonal or cyclic variation. We also did not stratify by drugs, analyzing marginal event reporting frequencies. Specific drugs or groups of drugs might be subjected to more or less seasonal or cyclic variation, and strategies for increasing power may facilitate such analysis. We also note the possibility of culture reporting differences/biases; and, of course, there is the influence that unusual extreme weather changes can have in the reporting patterns from one year to the next. We analyzed events for which seasonality in reporting makes sense.

Studying additional events, including those with less apparent logical connection to seasonality, may mean the discovery of similar yet unexpected reporting patterns that may refine pharmacovigilance signal detection, evaluation, and management. The same can be said of studying additional geographic regions. We analyze data from regions that are all in the Northern Hemisphere. But our method can be used to extend the analysis to include data from different hemispheres. For example, if we were analyzing data from the US and Australia, then all we need to do is shift one of the monthly datasets by 6 months. Thus, for example, the ordered list for the US could be, as usual, (Jan, Feb, Mar, ..., Dec) and that for Australia would then be (Jul, Aug, Sep, ..., Jun). Then the corresponding seasons would match: summer in the US (Jun, Jul, Aug) would correspond to summer in Australia (Dec, Jan, Feb); autumn in the US (Sep, Oct, Nov) would match autumn in Australia (Mar, Apr, May), etc.

\section{Conclusions}

Our preliminary study of a limited convenience sample suggests that performing quantitative analysis to detect seasonality of spontaneous adverse event reporting has the potential to usefully increase situational awareness in pharmacovigilance. As is often the case, the interwoven complexity of drug use and reporting behavior, and qualitative and quantitative limitations inherent in SRS data, may conspire against obtaining clear and consistent results in all cases. A natural extension of this work is using sets of test events that more fully accommodate the quantitative and phenotypic diversity of SRS data, as well as the structure of the dictionaries used to memorialize the data.

Author contributions Manfred Hauben conceived of the study. Eric Hung performed the data extraction/formatting. Osvaldo Marrero devised and performed the statistical analysis. All authors contributed substantially to the design of the study, and the writing, quality checking, and editing of the manuscript.

\section{Compliance with Ethical Standards}

Funding No sources of funding were used to assist in the preparation of this study.

Conflict of interest Manfred Hauben and Eric Hung are employees and stockholders of Pfizer, Incorporated. Osvaldo Marrero, Manfred Hauben, and Eric Hung have no other conflicts of interest that are relevant to the content of this study.

Open Access This article is distributed under the terms of the Creative Commons Attribution-NonCommercial 4.0 International License (http://creativecommons.org/licenses/by-nc/4.0/), which permits any noncommercial use, distribution, and reproduction in any medium, provided you give appropriate credit to the original author(s) and the source, provide a link to the Creative Commons license, and indicate if changes were made.

\section{References}

1. Lin M, Wang J, Chiu S, Chen C, Lue H, Wu M. Epidemiological evidence of seasonality in Kawasaki disease in Taiwan. ACTA CARDIOLOGICA SINICA. 2007;23(1):29.

2. Christensen AL, Rasmussen LH, Baker MG, Lip GY, Dethlefsen C, Larsen TB. Seasonality, incidence and prognosis in atrial fibrillation and stroke in Denmark and New Zealand. BMJ Open. 2012;2(4):e001210.

3. US Food and Drug Administration. FDA adverse event reporting system (FAERS). http://www.fda.gov/Drugs/GuidanceCompliance RegulatoryInformation/Surveillance/AdverseDrugEffects/default. htm. Accessed 4:04 pm 8 Feb 2015.

4. Bousquet C, Lagier G, Lillo-Le Louët A, Le Beller C, Venot A, Jaulent M-C. Appraisal of the MedDRA conceptual structure for describing and grouping adverse drug reactions. Drug Saf. 2005;28(1):19-34

5. Pearson RK, Hauben M, Goldsmith DI, Gould AL, Madigan D, O'Hara DJ, Reisinger SJ, Hochberg AM. Influence of the MedDRA ${ }^{\circledR}$ hierarchy on pharmacovigilance data mining results. Int J Med Inf. 2009;78:e97-103.

6. Edwards JH. The recognition and estimation of cyclic trends. Ann Hum Genet. 1961;25:83-6.

7. Marrero $\mathrm{O}$. L'analyse de la variation saisonnière quand l'amplitude et la taille sont faibles. Rev Can Statist (Can J Statist). $1999 ; 27: 875-82$.

8. Marrero O. A test statistic whose derivation is simple and unusual. Elem Math. 2001;56:157-62.

9. Olson AL, Swigris JJ, Raghu G, Brown KK. Seasonal variation: mortality from pulmonary fibrosis is greatest in the winter. Chest. 2009;136(1):16-22.

10. Marrero O. Multigroup analysis of seasonal variation: assessing the homogeneity of multiple cyclically ordered multinomial distributions. Environmetrics. 1998;9:151-63. 
11. Marrero O. Seasonal variation in epidemiology. Coll Math J. 2013;44:386-98.

12. El Marrero O. análisis de la variación estacional en medicina. Rev Mat Teoría y Aplicaciones. 2005;12:1-12.
13. Pierfitte C, Royer RJ, Moore N, Bégaud B. The link between sunshine and phototoxicity of sparfloxacin. Br J Clin Pharmacol. 2000;49:609-12. 